\title{
Focus on neurogenomics
}

\author{
Nature Neuroscience presents a series of Perspectives and Reviews highlighting recent advances in understanding \\ the genetics of complex brain disorders.
}

$\mathbf{P}$ reviously intractable to the approaches of human genetics, disorders of the brain are seeing accelerated gene discovery that was, until now, restricted to other branches of medicine. The driving force behind this progress is recent technological and analytical innovations that allow the interrogation of genetic variation at unprecedented resolution and scale and diminishing costs. With these plus collaborations among international consortia needed to reach adequate sample sizes, the past few years have suggested that robust gene discovery is possible for brain disorders. What is starting to emerge from these recent advances is that the genetic architecture of common brain disorders is exquisitely complex and heterogeneous. Translating these discoveries into a better understanding of disease etiology and the generation of new drug targets represent important challenges for neuroscience research. In this Focus on neurogenomics, we have compiled a series of Perspectives and Reviews on the success of genome-wide association and wholeexome sequencing studies in identifying genetic variants associated with neurodevelopmental, neurological and psychiatric disorders, which we hope will catalyze future endeavors to decipher and cure brain diseases.

Human genetics now has at its disposal a variety of approaches for identifying risk variants across the whole genome. In their Perspective on page 756, McCarroll, Feng and Hyman provide a primer on genomewide approaches and how these may be translated into mechanistic understanding of disease. They suggest that current translational attempts must be complemented by approaches that have far higher throughput and that permit analysis of biological systems beyond a single hypothesis involving a lone risk allele.

Disease risk for complex disorders involves multiple alleles that vary widely in population frequency. The Review on page 782 by Gratten, Wray, Keller \& Visscher discusses the genetic architecture of psychiatric disorders and the contribution of common and rare variants to disease risk. The etiological heterogeneity of these disorders is vast and complex, and a more complete picture of genetic risk is needed to understand other contributing causal factors. Following on the heels of genome-wide association studies, sequencing studies are beginning to provide potential clues about rare and new (de novo) mutations. But given that even healthy individuals carry many potentially deleterious mutations, how is disease causality assigned to rare variants found in patients?

In their Review on page 764, Hoischen, Krumm and Eichler discuss the statistical, clinical and biological factors that should be taken into account when prioritizing potential disease genes and assessing their causal contribution. They point out that there are now hundreds of new putative candidate genes that have been implicated in neurodevelopmental disorders such as autism spectrum disorders, intellectual disability and schizophrenia. The decision of which genes to pursue for further biological study will depend on many factors, including their mutational burden among healthy individuals, the involvement of the gene in disease-implicated protein networks and evidence of overlap with pathogenic copy number variants.

Understanding the clinical presentations of these diverse mutations presents its own puzzle. On page 773, Zhu, Need, Petrovski and Goldstein provide an analysis of Mendelian disease genes that highlights the difficulty in disentangling the mechanisms behind genotype-phenotype relationships in more complex diseases. They point out that it will be very challenging to unravel the causes of nonspecific risk and that multiple mechanisms may be involved. The authors suggest that, ultimately, to more fully understand phenotypically promiscuous mutations, we will need to resort to functional modeling in different genetic backgrounds.

All risk variants for psychiatric and neurological disorders must ultimately affect the functioning of the brain, and elucidating the affected neural circuits in humans is a high priority. In addressing this challenge, Medland, Jahanshad, Neale \& Thompson on page 791 summarize efforts of world-wide consortia that combine genome-wide studies with neuroimaging. They discuss the statistical and methodological issues necessary to insure rigor and robustness and avoid the pitfalls that beset the early days of imaging genetics.

A common thread in this Focus is the emphasis on statistical strength. As in other fields, human genetics has learned a hard lesson when it comes to low replication rates stemming from small, underpowered studies. The early days of psychiatric genetics saw a boom in candidate genes, but given how little is known about the underlying biology of most brain disorders, choosing viable candidates a priori is often highly subjective. Genetic association studies, genome wide or otherwise, need to be methodical and rigorous at the stages of study design, data collection and analysis. As researchers follow up on these associations, mechanistic dissection of genetic risk variants should ideally be as thorough and systematic as the methods used to discover them. As McCarroll et al. point out, this requires additional resources such as complete cell type-specific gene expression data from humans and appropriate high-throughput assays. Model systems should be tailored to the biological question asked and not used to recapitulate disease diagnoses of dubious biological validity. Once the relevant circuits and cell types are identified, the full arsenal of neuroscientific tools will likely be needed to understand their basic biology.

The ultimate goal of genomics is more effective treatments and perhaps preventative measures. This road from gene discovery to drug target, however, is arduous even for well-understood diseases with known pathophysiologies. With the realization that variants in hundreds of genes contribute to common brain disorders, delineating their point of convergence is a formidable challenge, and there is disagreement on which variants to focus on and how to best translate them into disease mechanisms and potential therapeutic targets. We hope this Focus will give our readers a glimpse into recent advances in neurogenomics and their potential for tangible neurobiological and therapeutic insights. 